\title{
Surface Normal Vector Force Driven 3D Object Reconstruction via Graph-cut
}

\author{
Sujung Kim and Seong Dae Kim \\ Department of Electrical Engineering \\ KAIST \\ Daejeon, Republic of Korea \\ sjkim@sdvision.kaist.ac.kr and sdkim@ee.kaist.ac.kr
}

\begin{abstract}
Multi-view 3D surface reconstruction is typically formulated as minimizing an appropriate energy functional, which tends to find the empty set, i.e. an undesirable result, especially when cooperating only with a boundary term. So generally a regional term is employed, which indicates inside or outside of the surface. It is, however, the challenge because inherently there is no straightforward way to assign interior or exterior of the surface since photo-consistency on the surface boundary is the main source of geometric information. In this paper, we address this problem by proposing a surface normal vector force driven regional term which is a data-aware ballooning term and cast it into a Graph-cut framework. In experiments, we verify the superiority of the proposed algorithm on various multiple view data sets.
\end{abstract}

Keywords-3D object reconstruction; multi-view stereo; graphcut framework; data-aware ballooning term; surface orientation;

\section{INTRODUCTION}

3D object reconstruction from multiple images captured from different viewing directions is one of the persistent issues in computer vision. It is typically formulated as minimizing an appropriate energy functional over a data term and a prior term. This approach has been advanced in this field quantitatively and qualitatively as described in [7]. The main drawback of minimizing a certain functional, however, is it tends to find the empty set, which is an undesirable result.

In order to prevent the shrinking behavior of the minimal surface model, a constant ballooning term is added in several approaches [8], [4]. Although it excludes the empty set, it makes the final reconstruction biased to the silhouette consistent shape, thus hard to find deep concavities. To overcome this side effect, a data-aware ballooning term was proposed by utilizing surface orientation estimated from the gradient of photo-consistency [1]. Though it opens up the use of a dataaware regional term instead of a constant ballooning term, it could not show acceptable results since the photo-consistency is likely to be erroneous for being used as surface orientation. Recently, the more elaborate regional term was proposed based on stereo information, which determines inside or outside by measuring the maximal photo-consistency along the viewing ray on every voxel [5]. It yields superior reconstruction results, but it takes high computational costs since every single ray through a voxel needs to be computed to find where a maximal point is.

In this paper, we propose a novel data-aware regional term, which takes account of surface normal vectors on reliable points cooperating with 3D Gaussian kernels. By employing reliable surface orientation, the proposed algorithm could find concavities well without computing every single ray per voxel to assign interior or exterior. In addition, it works well even with a small number of input images, where photo-consistency might not be densely available on surface boundary. We cast it into a Graph-cut framework which is well known to find a global minimal within polynomial time [6] and demonstrate the superiority of the proposed algorithm. This paper is organized as follows: we present the surface normal vector driven regional term in Section 2, and then Section 3 describes the 3D object reconstruction algorithm we used. Finally experimental results and conclusion are presented in Section 4.

\section{KERNEL BASED SURFACE NORMAL VECTOR FORCE}

\section{A. Definition}

The motivation of utilizing surface orientation is that it contains somewhat neighboring information of a 3D point, so it involves the associated shape of surface. In addition, surface orientation directly indicates the direction outward to the surface, which can be used to assign an inside or outside cost for a regional term.

The surface normal vector force on each 3D point, $\mathbf{x}$, is defined by

$$
\mathbf{f}(\mathbf{x})=\sum_{\mathbf{x}^{\prime} \in K_{s}} \mathbf{f}_{\mathbf{x}^{\prime}}(\mathbf{x})
$$

where $K_{s}$ indicates the set of reliable neighboring points determined by the Gaussian kernel size. Individual $\mathbf{f}_{\mathbf{x}^{\prime}}(\mathbf{x})$ is generated with a 3D Gaussian kernel as shown in Fig. 1, which is denoted by

$$
\mathbf{f}_{\mathbf{x}^{\prime}}(\mathbf{x})=\left(1-\rho\left(\mathbf{x}^{\prime}\right)\right) \cdot g_{\mathbf{x}^{\prime}}(\mathbf{x}) \cdot \mathbf{N}_{\mathbf{x}^{\prime}}(\mathbf{x}),
$$

where $\mathbf{x}^{\prime}$ is a reliable point and $\rho\left(\mathbf{x}^{\prime}\right)$ is its photo-consistency. The Gaussian function and the normal vector are described in (3) and (4).

$$
\begin{gathered}
g_{\mathbf{x}^{\prime}}(\mathbf{x})=e^{-\frac{\left\|\mathbf{x}-\mathbf{x}^{\prime}\right\|_{2}^{2}}{2 \sigma^{2}}} \\
\mathbf{N}_{\mathbf{x}^{\prime}}(\mathbf{x})= \begin{cases}\mathbf{N}\left(\mathbf{x}^{\prime}\right) & \text { if } \mathbf{x} \in \mathbf{B}_{\text {in }} \\
-\mathbf{N}\left(\mathbf{x}^{\prime}\right) & \text { if } \mathbf{x} \in \mathbf{B}_{\text {out }}\end{cases}
\end{gathered}
$$

In this formula, we need to know surface orientation in advance, so we used surface orientation estimated from the visual hull which is the general initialization in $3 \mathrm{D}$ object reconstruction algorithms. 


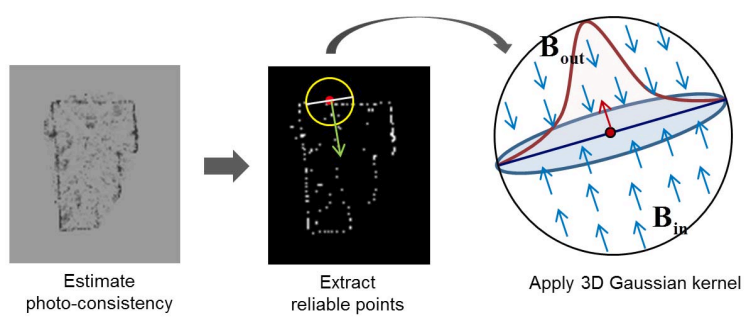

Fig. 1. Slice cut of a 3D object: 3D Gaussian kernel is applied on every reliable 3D points according to the amount of reliability to generate surface normal vector force.

\section{B. Cast in energy function}

We present a novel energy functional by employing the aforementioned surface normal vector force, $\mathbf{f}(\mathrm{x})$, to a regional term by

$$
E(S)=\int_{S} \rho(\mathbf{x}) d S-\int_{V(S)} \operatorname{sgn}(\mathbf{x}) \cdot|\mathbf{f}(\mathbf{x})| d V,
$$

where $S$ is the boundary between interior and exterior of the estimated surface, and $V(S)$ denotes the interior volume surrounded by $S$. The sign function is denoted by

$$
\operatorname{sgn}(\mathbf{x})= \begin{cases}1 & \text { if } \mathbf{f}(\mathbf{x}) \cdot \mathbf{N}(\mathbf{x})>0 \\ -1 & \text { if } \mathbf{f}(\mathbf{x}) \cdot \mathbf{N}(\mathbf{x})<0 .\end{cases}
$$

It implies that a $3 \mathrm{D}$ point is assumed to be located inside the surface if its surface normal vector force has the same direction with the original surface normal vector. Otherwise it considered outside. It is based on the assumption that surface orientation is rarely to be changed to the opposite direction of an initial surface, e.g. visual hull.

\section{3D OBJECT RECONSTRUCTION}

The flow diagram of 3D object reconstruction algorithm is described in Fig. 2. We generated an initial visual hull by [3], and used the publicly available source code for the graph-cut optimization in [6].

\section{RESULT AND CONCLUSION}

We tested our proposed algorithm on the dataset provided in [2], which contains 24 views of Nskulla $(1900 \times 1700)$ and Dinosaur $(2000 \times 1500)$ with calibrated camera projections. We used $512^{3}$ of the spatial voxel resolution in all experiments. The parameters with regard to the 3D Gaussian kernel are fixed: the kernel size, $s^{3}$, is $7^{3}$ and variance, $\sigma$, is equal to $0.6 \times s$. The patch size for computing photo-consistency is $15 \times 15$ and the threshold for extracting reliable points is set to 0.5 in all experiments.

Fig. 3 shows the reconstruction results with the visual hull results. Compared with initializations, our proposed algorithm represents the concavities well, e.g. the eye and nose of the
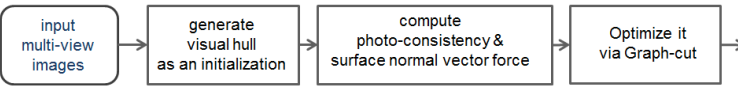

Fig. 2. Flow diagram of 3D object reconstruction algorithm

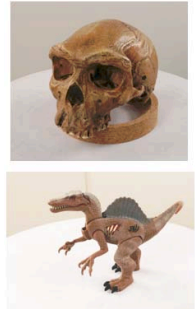

(a)

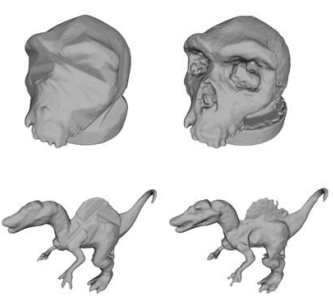

(b)

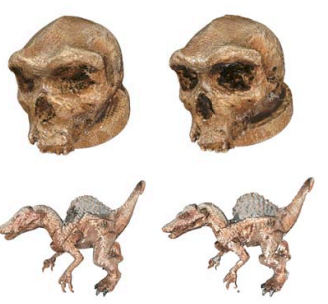

(c)
Fig. 3. Reconstruction results. (a) one of input images. (b) geometric results from the visual hull (left) and the proposed method (right) (c) reconstruction with texture corresponding to (b).

skull and the face and back of the dinosaur. The computational time is approximately 15 and 7 minutes respectively, which is considerably fast compared to the state of the art methods [2][5].

In this paper, we proposed the 3D object reconstruction algorithm based on the data-aware regional term, which is driven by surface normal vector forces. And also we cast it into a Graph-cut framework which is known to find a global minimal. Therefore it could reconstruct concavities well within a reasonable computational time compared to other methods.

\section{ACKNOWLEDGMENT}

This research was supported by Mid-career Researcher Program through NRF grant funded by MEST.

\section{REFERENCES}

[1] Y. Boykov and V. Lempitsky. From photohulls to photoflux optimization. In British Machine Vision Conference, pages 1-10, 2006.

[2] Y. Furukawa and J. Ponce. Accurate, dense, and robust multi-view stereopsis. IEEE Transactions on Pattern Analysis and Machine Intelligence, $32(8), 2010$

[3] S. Kim, H. Kim, W. Kim, and S. Kim. Fast computation of a visual hull. In Asian Conference of Computer Vision, pages 1-8, 2010.

[4] K. Kolev, M. Klodt, T. Brox, and D. Cremers. Propagated photoconsistency and convexity in variational multiview $3 \mathrm{~d}$ reconstruction. In Workshop on photometric analysis for computer vision, 2007.

[5] K. Kolev, M. Klodt, T. Brox, and D. Cremers. Continuous global optimization in multiview $3 \mathrm{~d}$ reconstruction. International Journal of Computer Vision, 84:80-96, 2009.

[6] V. Kolmogorov and R. Zabih. What energy functions can be minimized via graph cuts? IEEE Transactions on Pattern Analysis and Machine Intelligence, 26:147-159, 2004.

[7] S. Seitz, B. Curless, J. Deiabel, D. Scharstein, and R. Szeliski. A comparison and evaluation of multi-view stereo reconstruction algorithms. In IEEE Computer Vision and Pattern Recognition, pages 519-528, 2006.

[8] G. Vogiatzis, C. Hernandez, P. Torr, and R. Cipolla. Multiview stereo via volumetric graph-cuts and occlusion robust photo-consistency. IEEE Transactions on Pattern Analysis and Machine Intelligence, 29(12), 2007. 